\title{
Research about the Degree of Relation between Chaotic Characteristics of Physiological Signals and Emotions
}

\author{
Nie Chun yan ${ }^{1, \mathrm{a}}$, He Fang ${ }^{2, \mathrm{~b}}$, Xia Ying ${ }^{1, \mathrm{c}}$ and Wang Ju ${ }^{1, \mathrm{~d}}$ \\ ${ }^{1}$ Electronic Information Institute Changchun University, China \\ ${ }^{2}$ School of Electronics and Information Engineering, Changchun University of \\ Science and Technology, Changchun 130022, China \\ ncy66@163.com, ${ }^{b}$ hefang0724@sina.cn, \\ ${ }^{c} 18244008351 @ 163 . c o m,{ }^{d}$ youyou8221@yahoo.com.cn
}

\begin{abstract}
Physiological information can objectively reflect the emotional state. Emotion recognition based on physiological signals has the important practical application value. In this paper, six kinds of chaotic characteristics were extracted from physiological data about Electromyogram, Skin conductivity, Respiration, which come from Augsburg university in Germany. Six kinds of chaotic characteristics contain the largest Lyapunov exponent, correlation dimension, box dimensions, information entropy, approximate entropy and complexity. On the basis of this, the degree of relation between chaotic characteristics of physiological signals and emotions was researched. The results showed that different chaotic characteristic values of different physiological signals sensitive to the emotion is different. Therefore, in order to improve the recognition rate, the chaotic characteristics of the multiple physiological information need to be merged to establish chaos matrix, which provide a new method for emotion recognition.
\end{abstract}

Keywords: physiological signal, chaotic characteristic, emotion recognition

\section{Introduction}

Domestic and overseas scholars in the study of physiological signal to extract the feature parameter method each are not identical, previous targets is based on linear feature. In this paper, the extraction of the chaotic characteristics was studied based on the nonlinear chaos theory. Presently, the main physiological signals were used in the study of emotion recognition, which including Electrocardiogram (ECG), Electromyogram (EMG), Skin conductivity(SC), Respiration(RSP), Electro-Oculogram(EOG), Electroencephalogram (EEG), ect. Physiological signals in this thesis are ECG, EMG, SC, RSP.

\section{Selection of Physiological Signals}

\subsection{Electrocardiogram}

Electrocardiogram (ECG) signal is potential difference formed on the pleural surface when the heart beats. When the patch electrode is attached to a specific location near the heart, the ECG is obtained by measuring the wire connection to the instrument to record the change of potential difference. Ekman and others found when heart rate of people reached the highest in fear, anger, happy, however, the heart rate slows down in sorrow, while in disgust the heart rate is the lowest [1]. So ECG can reveal mood changes, which can extract the characteristic parameters of ECG signal to identify the emotion. 


\subsection{Electromyogram}

Electromyogram (EMG) signal is the sum of the action potentials of muscle contraction or expansion. The EMG signal is the electrical signal generated from muscle activities by the instrument of measurement, which belong to positioned at the muscle's wire to connect [2]. In different emotional states, muscles of people will produce a corresponding shrinkage or expansion. For example, when people are in angry, fingers will be clenched, muscles between the neck and arm will shrink, EMG becomes larger. So EMG can reveal mood changes, and extracting the characteristic parameters of EMG signal can identify the emotion.

2.2.3. Skin Conductivity: Skin conductivity (SC) signal represents the conductivity of the skin. The two fingers are connected with a sensitive response of electricity meter by measuring wires. When table pointer wavers, the potential difference measured is called SC signals. The change of SC signals is closely related to people's emotional state changed. For example, people in sadness, the vein shrink, sweat secretion reduced, skin conductance decreased. However, when people are scared, sweat secretion increased, and skin conductance increased. All of this leads to the change of SC signal. So SC can reveal mood changes. The emotion is identified by extracting the characteristic parameters of SC signal.

2.2.4. Respiration: Respiratory (RSP) signals are composed of the respiratory amplitude and breathing rate the during expansion motion of thoracic cavity. The elastic nylon belt which has a sensor is wound around the chest in the human body. In the process of breathing, by measuring the telescopic amount of breath strap which shows voltage increasing or decreasing, thus obtaining RSP signal. RSP signals are very important to identify the emotional state. It's change will combined with the change of emotion state. For example, people are in a happy, anger or fear, people breathing tend to be deep and fast breathing. When in a tense, people breathing always is superficial and rapid. So RSP can reveal mood changes, which can identify the emotion by extracting the characteristic parameters of RSP signal .

\section{The Source of the Physiological Data}

Johannes Wagner, et al, who came from Augsburg University in Germany broadcasted four different key tone music to the testers, making them in joy, anger, sadness and pleasure in four kinds of different emotional state. Under each kind of mood, signals of ECG, EMG, SC and RSP were separately collected within 2 minutes, thus establishing emotional physiological databases. Data collection lasted 25 days, so four kinds of emotions respectively have 25 samples, namely, the total of samples is 100 . The emotional physiological database of Augsburg University in Germany is an influential and authoritative database used more in computing field. A lot of physiological signals researched by domestic scholars in emotion recognition come from this database. In order to compare their research results with those of related scholars, in this paper, use the date as experimental data of physiological signals in chaotic Characteristics extraction.

\section{Extraction of Chaotic Characteristics Parameters}

Dealing with biomedical signals based on nonlinear chaotic theory is the focus of research in recent years. Extracting the chaotic characteristic parameters of the physiological information opens up a new direction to the research of emotion recognition. Six kinds of chaos characteristics are extracted in this thesis, which are the largest Lyapunov exponent, correlation dimension, fractal dimensions, 
information entropy, approximate entropy and complexity, which provides a new method to improve emotion recognition rate.

\subsection{Extraction of the Largest Lyapunov Exponent}

Lyapunov exponent is a parameter used to represent the influence of initial conditions to chaotic motion, and which is an important parameter of quantitative expression to the chaotic systems. Wolf pointed out using the phase trajectory, phase plane, phase volume method to estimate of the Lyapunov exponent, which is uniformly called Wolf method ${ }^{[3]}$.

The largest Lyapunov exponents in the state of Joy, Anger, Sadness and Pleasure are obtained by Wolf method to calculating the date in Augsburg emotional physiological database about ECG, EMG, SC, RSP, as shown in Table 1.

It can be found through analysis, that the largest Lyapunov exponents of ECG under different emotions appear the overlapping values, which can not accurately distinguish emotion between four kinds of emotions. Particularly in the pleasure emotion, the largest Lyapunov exponents of ECG signals have the largest span. The largest Lyapunov exponents of EMG and SC in different emotion also appear the overlapping values, which can not accurately distinguish emotion between four kinds of emotion. However, in the Joy and Sadness emotions, the largest Lyapunov exponents don't appear overlapping. The largest Lyapunov exponents of RSP signals in the Joy mood are not overlapping with those of other three emotions, by which the Joy emotion can be easily distinguished. Although this, it is difficult to distinguish emotions.

Table 1. Largest Lyapunov Exponents of Four Kinds of Physiological Signals

\begin{tabular}{ccccc}
\hline & ECG & EMG & SC & RSP \\
\hline Joy & $0.0144 \sim 0.1023$ & $0.0990 \sim 0.9337$ & $0.0172 \sim 0.1564$ & $0.0084 \sim 0.0105$ \\
Anger & $0.0102 \sim 0.1023$ & $0.0699 \sim 0.8862$ & $0.0914 \sim 0.2093$ & $0.0212 \sim 0.0891$ \\
Sadness & $0.0135 \sim 0.1020$ & $0.1687 \sim 1.5613$ & $0.1638 \sim 0.2156$ & $0.0864 \sim 0.1163$ \\
Pleasure & $0.0041 \sim 0.1761$ & $0.0115 \sim 0.6136$ & $0.0111 \sim 0.1781$ & $0.0170 \sim 0.1005$ \\
\hline
\end{tabular}

\subsection{The Extraction of Correlation Dimension}

The category of the fractal dimension contain box dimension, correlation dimension, information dimension and so on. The application of correlation dimension and box dimension are relatively extensive. The correlation dimension represents the correlation degree among the phase points in the system, which is a very important characteristic parameter of the chaotic nonlinear system. P.Grassberger construct the G-P algorithm to calculate the correlation dimension based on the idea of delay embedding space [4]. This algorithm is simple to be implemented and easy to be understood.

The correlation dimensions of the ECG signals are extracted about four emotions in first 10 days in connection with the data of the emotion physiology database. We find that the correlation dimensions of ECG signals are not integers, which show the ECG are nonlinear signals with chaotic characteristics, and there are strange attractors, the trajectory of which is complex. The correlation dimension values of ECG, EMG, SC and RSP signals are obtained by using G-P algorithm in Joy, Anger, Sadness and Pleasure emotions. The correlation dimension values of four kinds of physiological signals are shown in Table 2 .

Table 2. Correlation Dimensions of Four Kinds of Physiological Signals

\begin{tabular}{llll}
\hline ECG & EMG & SC & RSP
\end{tabular}




\begin{tabular}{ccccc}
\hline Joy & $0.7380 \sim 1.8732$ & $0.1983 \sim 4.9568$ & $1.9686 \sim 2.8718$ & $0.3648 \sim 0.7281$ \\
Anger & $0.5242 \sim 1.9126$ & $0.6845 \sim 5.0391$ & $1.7163 \sim 2.8992$ & $0.0412 \sim 0.7819$ \\
Sadness & $0.7175 \sim 2.2938$ & $0.2122 \sim 3.7842$ & $1.9005 \sim 3.6805$ & $0.9186 \sim 1.1633$ \\
Pleasure & $0.5543 \sim 2.2733$ & $0.1595 \sim 3.8443$ & $1.8016 \sim 2.6639$ & $0.0170 \sim 0.4005$ \\
\hline
\end{tabular}

It can be found through analysis, that the correlation dimensions of ECG, EMG and SC in different emotions appear the overlapping values, which can not accurately distinguish emotion between four kinds of emotions. In the Sadness emotion, the range of the correlation dimensions of RSP signals are not overlapping with those of other three emotions, by which the Sadness emotion can be easily distinguished. But, the correlation dimensions of Joy, Anger and Pleasure are overlapping.

\section{3. The Extraction of Chaos Box Dimension}

The Box dimension can well describe the complexity and irregularity of time series, and can be easily completed by computer, so, there is very wide application. N. Sarkar and B. Chaudhuri proposed DBC (Differentia Box Couting) algorithm in 1994 [5]. The algorithm is fast, convenient and accurate.

Let $A$ is the set of n-dimensional Euclidean space $R^{n}, F \subset A$ and let $N_{\varepsilon}(F)$ is the side containing subset $F$, and is minimum number of n-dimensional cube $\varepsilon$, so, the box dimension of $F$ is

$$
D_{B}=\lim _{\varepsilon \rightarrow 0} \frac{\lg N_{\varepsilon}(F)}{-\lg \varepsilon}
$$

The specific calculation steps are as follows:

(1) Determine the length of the maximum edge $\varepsilon$, which is even power of two $(1,2,4,8$ ...).

Selecting the even greater than the length of data. Because the length of sample data is 3000 , so, $\varepsilon=16$;

(2) Preprocessing on the physiological signals and normalized to the unit square range, so

$F=\{x(t) \mid \min (x(t))=0, \max (x(t))=1,0 \leq t \leq 1\}$

(3) With in the range of $I_{n}=[(n-1) \varepsilon, n \varepsilon]$, calculating the least square number $N_{\varepsilon}(F)$, which cover the subset

Where, $N_{n}=[(\max (x(t))-\min (x(t))) / \varepsilon]$

$N_{\varepsilon}(F)=\sum_{n=1}^{1 / \varepsilon} N_{n}$

(4) Box dimension

$D_{B}=\lim _{\varepsilon \rightarrow 0} \frac{\lg N_{\varepsilon}(F)}{-\lg \varepsilon}$ 
The box dimensions of ECG, EMG, SC and RSP signals are obtained by using DBC algorithm, in Joy, Anger, Sadness and Pleasure emotions. The box dimension values of four kinds of physiological signals are shown in Table 3.

Table 3. The Box Dimension Values of Four Kinds of Physiological Signals

\begin{tabular}{ccccc}
\hline & ECG & EMG & SC & RSP \\
\hline Joy & $1.3327 \sim 1.4496$ & $1.3387 \sim 1.6731$ & $1.1564 \sim 1.5276$ & $1.2918 \sim 1.3678$ \\
Anger & $1.3376 \sim 1.4375$ & $1.5111 \sim 1.6840$ & $1.1441 \sim 1.6153$ & $1.3710 \sim 1.4586$ \\
Sadness & $1.3204 \sim 1.4475$ & $1.3759 \sim 1.6309$ & $1.2076 \sim 1.5516$ & $1.3798 \sim 1.4045$ \\
Pleasure & $1.3192 \sim 1.4463$ & $1.4332 \sim 1.6458$ & $1.1698 \sim 1.4784$ & $1.3986 \sim 1.4238$ \\
\hline
\end{tabular}

It can be found through analysis, that the box dimensions of ECG, EMG and SC in different emotions appear the overlapping values, which can not accurately distinguish emotions between four kinds of emotions. In the Joy emotion, the ranges of the box dimension of RSP signals are not overlapping with those of other three emotions, by which the Joy emotion can be easily distinguished. But, the box dimensions of Anger, Sadness and Pleasure are overlapping.

\subsection{Extraction of Information Entropy}

Information entropy represents the uncertainty of the information source, namely disorder [6]. The system is more chaotic, the value of the information entropy is greater. Conversely, the system is ordered, the information entropy value is smaller.

For discrete sequences, the information entropy is expressed as follows:

$H(X)=\sum_{x \in \operatorname{dom}(X)} p(x) \log p(x)$

Where, $\operatorname{dom}(X)$ is the selected range of $X$.

The experiment data of physiological signals are divided into segmentation. Next, the information entropy values of each section are calculated. Finally, the total information entropy values are obtained by superposition. Each sample is 3000 points. Experimental data obtained is divided into 10 segments through the optimization algorithm. The information entropy values of ECG, EMG, SC and RSP signals are obtained by using relational definition algorithm in Joy, Anger, Sadness and Pleasure emotions. The Information entropy values of four kinds of physiological signals are shown in Table 4.

Table 4. Information Entropies of Four Kinds of Physiological Signals

\begin{tabular}{ccccc}
\hline & ECG & EMG & SC & RSP \\
\hline Joy & $1.2016 \sim 2.0254$ & $0.0456 \sim 2.6763$ & $1.5912 \sim 3.1235$ & $2.5383 \sim 3.1909$ \\
Anger & $1.0597 \sim 1.8695$ & $1.2656 \sim 2.4937$ & $1.6044 \sim 3.2614$ & $2.5587 \sim 3.1552$ \\
Sadness & $1.0058 \sim 2.1071$ & $0.3654 \sim 2.1706$ & $2.0928 \sim 3.2954$ & $2.7362 \sim 3.2247$ \\
Pleasure & $1.1287 \sim 1.9072$ & $0.9116 \sim 2.7737$ & $1.8962 \sim 3.2759$ & $2.4705 \sim 3.1582$ \\
\hline
\end{tabular}

It can be found through analysis, that the information entropies of ECG, EMG, SC and RSP in different emotions appear the overlapping values, which can not accurately distinguish emotions between four kinds of emotions. In the Sadness emotion, the information entropy values of ECG signals have the largest span. In the Joy emotion, the information entropy values of ECG signals have the largest span. In the Pleasure emotion, the information entropy values of RSP signals are smaller. 


\subsection{The Extraction of Approximate Entropy}

Approximate entropy is the chaos characteristic parameters which measure the complexity of time sequences. The stable values are obtained by less data, which is more suitable for engineering applications. Approximate entropy values of chaotic systems are non-negative numbers. The higher the complexity of the system is, the greater the values of the approximate entropy are. Conversely, lower the complexity is, its values are smaller.

The approximate entropy values of ECG, EMG, SC and RSP signals are obtained by using relational definition algorithm in Joy, Anger, Sadness and Pleasure emotions. The approximate entropy values of four kinds of physiological signals are shown in Table 5.

Table 5. Approximate Entropy of Four Kinds of Physiological Signals

\begin{tabular}{ccccc}
\hline & ECG & EMG & SC & RSP \\
\hline Joy & $0.1451 \sim 0.6666$ & $0.3577 \sim 1.3316$ & $0.0213 \sim 0.5028$ & $0.1545 \sim 0.1656$ \\
Anger & $0.1314 \sim 0.6062$ & $0.3762 \sim 1.7242$ & $0.0641 \sim 0.3491$ & $0.3430 \sim 0.4398$ \\
Sadness & $0.1415 \sim 0.5371$ & $0.1430 \sim 1.2683$ & $0.0564 \sim 0.3248$ & $0.1693 \sim 0.1917$ \\
Pleasure & $0.1223 \sim 0.4626$ & $0.1982 \sim 1.8321$ & $0.0295 \sim 0.4713$ & $0.2270 \sim 0.2881$ \\
\hline
\end{tabular}

It can be found through analysis, that the approximate entropies of ECG, EMG, SC and RSP in different emotions appear the overlapping values, which can not accurately distinguish emotions between four kinds of emotions. In the Pleasure emotion, the approximate entropy values of ECG signals are smaller. In the Sadness emotion, the approximate entropy values of EMG signals are smaller. In the Joy emotion, the approximate entropies of SC signals have the largest span. In the Anger emotion, the approximate entropies of RSP are relatively large numerical.

\subsection{Extraction of the Complexity}

Lempel and J. Ziv proposed a specific algorithm which computed the complexity in 1976, namely, L-Z Complexity [7]. The complexity can quantitatively describe complex signals, which provide a kind of analysis method about time series. So, the complexity received great attention in the analysis of physiological signals.

The L-Z complexity values of ECG, EMG, SC and RSP signals are obtained by using definition algorithm in Joy, Anger, Sadness and Pleasure emotions. The complexity values of four kinds of physiological signals are shown in Table 6.

Table 6. The Complexity Values of Four Kinds of Physiological Signals

\begin{tabular}{ccccc}
\hline & ECG & EMG & SC & RSP \\
\hline Joy & $0.1078 \sim 0.1810$ & $0.2503 \sim 0.9048$ & $0.0116 \sim 0.2965$ & $0.1224 \sim 0.1656$ \\
Anger & $0.1155 \sim 0.2156$ & $0.1309 \sim 0.6930$ & $0.0193 \sim 0.4043$ & $0.1733 \sim 0.2272$ \\
Sadness & $0.2195 \sim 0.2310$ & $0.2464 \sim 0.5544$ & $0.0193 \sim 0.2233$ & $0.1040 \sim 0.1348$ \\
Pleasure & $0.1001 \sim 0.2657$ & $0.1848 \sim 0.7585$ & $0.0116 \sim 0.2349$ & $0.0943 \sim 0.1309$ \\
\hline
\end{tabular}

It can be found through analysis, that the complexity of ECG, EMG, SC and RSP in different emotions appear the overlapping values, which can not accurately distinguish emotions between four kinds of emotions. In the Sadness emotion, the complexity values of ECG signals are larger. In the Joy emotion, the complexity values of EMG signals are larger. In the Anger emotion, complexity values of RSP are relatively large numerical.

In summary, the degree of association is obtained between chaotic characteristics of physiological signals and different emotions. Through the analysis of the degree of association, the conclusion show the four kinds of emotions can not be clearly 
distinguished, by only one kind of chaotic characteristics, but, it need six kinds of chaos characteristic parameters composed together to distinguish four emotions.

\section{Establishment of Chaos Characteristic Matrix}

For four kinds of physiological signals, respectively to calculate the largest Lyapunov exponent, the correlation dimensions, the box dimension, complexity and approximate entropy and information entropy of every sample, on this basis, establish sample chaotic characteristic matrix is shown in Figure1, which is the preparation for the emotion recognition.

\section{Conclusion}

In this paper, six kinds of chaotic characteristics were respectively extracted from four kinds of physiological signals such as the largest Lyapunov exponent, correlation dimension, box dimension, information entropy, approximate entropy and complexity. After analysis, we find that different chaotic characteristic values of different physiological signals sensitive to the emotion is different. A single chaos characteristic can not completely recognize four kinds of emotion states. So, to recognize different emotions need to build chaotic characteristic matrix. The chaotic characteristic of the multiple physiological information were merged to solve the problem of recognition. With the development of nonlinear dynamics, the analysis method of nonlinear chaos theory can be expected to provide a new method for improving emotion recognition rate.

\begin{tabular}{|c|c|c|c|}
\hline \multirow{4}{*}{$\begin{array}{l}100 \\
\text { samples }\end{array}$} & $\begin{array}{c}\text { Joy } \\
25 \text { samples }\end{array}$ & \multicolumn{2}{|c|}{$\begin{array}{ll}\text { ECG、EMG、 } & \text { SC } 、 \text { RSP } \\
\text { Six chaotic characteristic } \\
\text { parameters }\end{array}$} \\
\hline & $\begin{array}{c}\text { Anger } \\
25 \text { samples }\end{array}$ & $\begin{array}{l}\text { ECG、EMG } \\
\text { Six chaotic } \\
\text { parameters }\end{array}$ & $\begin{array}{l}\mathrm{SC} 、 \mathrm{RSP} \\
\text { characteristic }\end{array}$ \\
\hline & $\begin{array}{c}\text { Sadness } \\
25 \text { samples }\end{array}$ & $\begin{array}{l}\text { ECG、EMG } \\
\text { Six chaotic } \\
\text { parameters }\end{array}$ & $\begin{array}{l}\mathrm{SC} 、 \mathrm{RSP} \\
\text { characteristic }\end{array}$ \\
\hline & $\begin{array}{c}\text { Pleasure } \\
25 \text { samples }\end{array}$ & $\begin{array}{l}\text { ECG、EMG } \\
\text { Six chaotic } \\
\text { parameters }\end{array}$ & $\begin{array}{l}\text { SC 、 RSP } \\
\text { characteristic }\end{array}$ \\
\hline
\end{tabular}

Figure 1. Chaotic Characteristic Matrix

\section{Acknowledgements}

This work is partially supported by The Ministry of education "Chunhui plan" Grant No. Z2014136 and Jilin Province Science and Technology Development Program Grant No. 20150204039SF. 
International Journal of $u-$ and $\mathrm{e}-$ Service, Science and Technology

Vol. 9, No. 12 (2016)

\section{References}

[1] P. Ekman, "The Science of On-Camera Acting", Becoming Media, vol. 9, (2014).

[2] L. Hao, "Research of surface electromyography collection system", Hebei University of Technology,(2010).

[3] A. Wolf, J. B. Swift and H. L. Swinney, "Time-Delay Systems: Lyapunov Functionals”, Birkhauser, (2012).

[4] P. Grassberger and I. Procaccia, "Characterization of strange attractors", Phys Rev Lett, vol. 346, (2003).

[5] B. Chaudhuri and N. Sarkar, "Texture image classification using multi fractal dimension", Journal of Marine Science and Application, vol. 2, (2003).

[6] C. M. DU, J. G. CUI and F. TIAN, "Calculation of the Information Entropy of EMG in Two-dimension Phase Space", Control Engineering of China, Supp. 1, (2006), pp. 107-109.

[7] J. Diaz, "Algorithms and Complexity", 7th International Conference, Italy, (2010), pp. 26-28. 